Chirurg 2013 $84: 433$

DOI 10.1007/s00104-013-2504-1

Online publiziert: 7. April 2013

๑) Springer-Verlag Berlin Heidelberg 2013
H. Dralle $\cdot$ I. Satiroglu

Universitätsklinik für Allgemein-, Viszeral- und Gefäßchirurgie,

Medizinische Fakultät, Universitätsklinikum Halle, Halle (Saale)

\title{
Laparoskopische Chirurgie neuroendokriner Pankreastumoren
}

\section{Originalpublikation}

Haugvik SP, Marangos IP, Rosok Bl et al (2013) Long-term outcome of laparoscopic surgery for pancreatic neuroendocrine tumors. World J Surg 37:582-590

\section{Hintergrund und Fragestellung}

Neuroendokrine Pankreastumoren (NEPT) haben einen Anteil von etwa $2-4 \%$ an allen klinisch diagnostizierten Pankreastumoren. Da mit den heute zur Verfügung stehenden bildgebenden Verfahren NEPT häufig in Tumorgrößen entdeckt werden, die prinzipiell für ein laparoskopisches Vorgehen geeignet sind, war Ziel der vorliegenden retrospektiven Single-Center-Studie die Evaluation des operativen Risikos und des Langzeitverlaufes nach laparoskopischer Resektion oder Enukleation.

\section{Methoden}

Von 1997 bis 2011 wurden nach ausgiebiger Bildgebung und Laborchemie 75 laparoskopische Resektionen/Enukleationen bei 72 Patienten mit NEPT durchgeführt. Zur exakten intraoperativen Tumorlokalisation und Lagebeurteilung hinsichtlich der Nähe zum Pankreasgang erfolgte bei allen laparoskopischen Operationen eine intraoperative Ultraschalluntersuchung. Sechs Patienten hatten eine Multipleendokrine-Neoplasie-1-Erkrankung.

\section{Ergebnisse}

In der berichtenden HPB (hepatopankreatobiliären) -Klinik wurden 75 der insgesamt 241 laparoskopischen Pankreaseingriffe (31\%) wegen NEPT durchgeführt. Bei 10 laparoskopischen Eingriffen erfolgte eine Konversion zur Laparotomie $(n=4 ; 5 \%)$, bei 6 lediglich eine Exploration. 51 laparoskopische Eingriffe waren distale Pankreasresektionen (79\%) ohne $(n=23)$ oder mit $(n=28)$ Splenektomie, in 14 Fällen erfolgte eine Enukleation im Kopfbereich $(n=7)$ oder Korpusschwanz $(\mathrm{n}=7)$. Bei 8 Patienten wurden Umgebungsorgane mitentfernt (Nebenniere, Leber, Magen, Kolon). Bei 2 Insulinompatienten war keine Tumorlokalisation im Rahmen der Laparoskopie möglich. 5 Patienten (8\%) wurden wegen postoperativer Komplikationen reoperiert, davon 4 erneut laparoskopisch. Die postoperative Fistelrate betrug nach Enukleation $50 \%$, nach Resektion 14\%, darunter keine Grad-C-Fistel. Die 5-Jahres-Überlebensrate lag bei $90 \%$, sie wurde bestimmt durch die T- und R-Kategorie sowie den Ki67-Index. Bei 22\% der Patienten war der histologische R-Status R1 oder R2.

\section{Fazit und Kommentar}

- Laparoskopische Resektionen oder Enukleationen bei NEPT sind mittlerweile in den meisten spezialisierten Abteilungen Bestandteil des chirurgischen Konzepts. Sie erfordern eine exakte prä- und intraoperative Bild- gebung mit laparoskopischem Ultraschall. Bei 2 Insulinompatienten konnte der Tumor dennoch nur durch Laparotomie gesichert werden.

- Die postoperative Fistelrate nach Enukleation war in der vorliegenden Untersuchung sehr hoch (50\%), allerdings ohne Grad-C-Fisteln.

- Bei $22 \%$ der Patienten erfolgte keine R0-Resektion, das laparoskopische Vorgehen war daher potenziell von Nachteil für das Langzeitüberleben.

Laparoskopische Enukleationen sollten nur dann durchgeführt werden, wenn das zu erwartende Fistelrisiko nicht höher liegt als beim offenen Vorgehen. Laparoskopische Operationen bei NEPT sind nicht zu empfehlen, wenn eine bei offener Operation vermeidbare R1-/R2Situation resultiert.

\section{Korrespondenzadresse}

Prof. Dr. Dr. h.c. H. Dralle

Universitätsklinik für Allgemein-, Viszeralund Gefäßchirurgie, Medizinische Fakultät, Universitätsklinikum Halle, Ernst-Grube-Str. 40, 06097 Halle (Saale) henning.dralle@uk-halle.de

Interessenkonflikt. Keine Angaben 